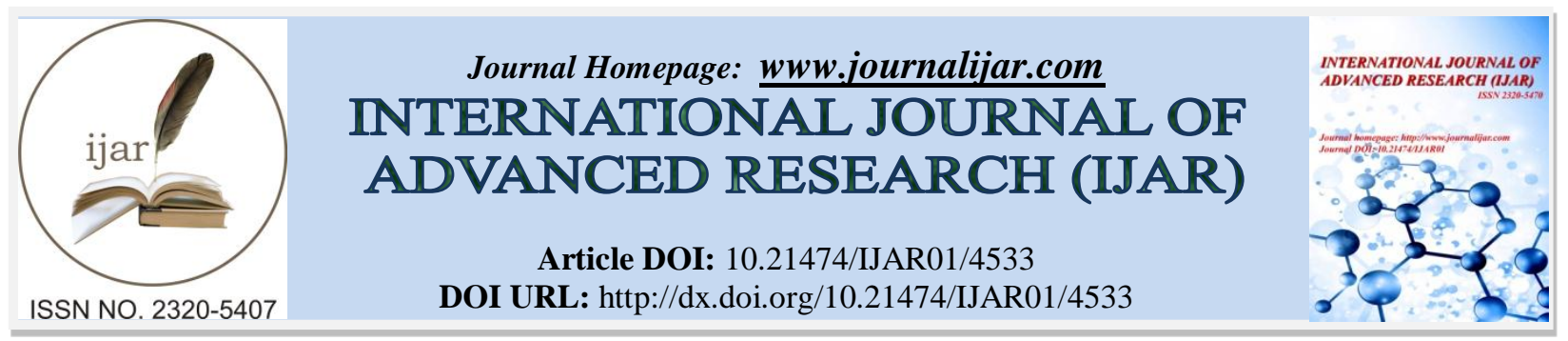

RESEARCH ARTICLE

\title{
EVALUATION OF RISK AND PROGNOSTIC FACTORS OF CARBAPENEM RESISTANT ACINETOBACTER BAUMANNII INFECTIONS IN INTENSIVE CARE UNIT.
}

Fulya Bayındır Bilman ${ }^{1}$, Barış Çiçek ${ }^{2}$ and Cem Ece ${ }^{3}$.

1. İzmir Menemen State Hospital, Department of Medical Microbiology, İzmir, Turkey.

2. İzmir Menemen State Hospital, Clinic of Infectious Disease, İzmir, Turkey.

3. İzmir Cigli Education and Research Hospital, Department of Anesthesiology and Critical Care Medicine, İzmir, Turkey.

\section{Manuscript Info}

\section{Manuscript History}

Received: 19 April 2017

Final Accepted: 21 May 2017

Published: June 2017

Key words:

Acinetobacter baumannii, ventilator associated pneumonia

\section{Abstract}

Objective: Multi-drug resistant Acinetobacter baumannii isolation is increasing among hospital infections. This study aims to examine the factors affecting the morbidity and mortality together with the selections and periods of the treatment applied on multi-drug resistant and extreme drug resistant $A$. baumannii infections that develop in intensive care unit patients.

Materials and Methods: Of the 20 cases involved in the study (10 male, 10 female; mean age 71,9 \pm 16 years), laboratory and treatment results as well were assessed during the treatment. Antibiotic susceptibility test was practiced with disk diffusion test in $A$. baumannii strains and it was practiced by using $\mathrm{E}$ test for colistin, meropenem, and imipenem.

Results: The infections detected as ventilator associated pneumonia (VAP) were determined on the 14th day of hospitalization on the average, and the follow up period is 40,8 days in the intensive care unit (ICU). The values of Acute Physiology and Chronic Health Evaluation II (APACHE II) was 26,8 $\pm 3,2$. The most preferred combinations are colistin-sulbactam and colistin-carbapenem. In three of the cases, the isolates were resistant to all antibiotics. During colistin treatment, dermatitis was observed in two subjects and nephrotoxicity in five subjects. Thirteen of the subjects were lost because of not eradicating the active microorganism resistant to antibiotics.

Conclusion: In our study it was concluded that early diagnosis and treatment and needed isolation precautions are important in infection follow-up in the infections caused by A. baumannii strains that have an important role in intensive care infections.

Copy Right, IJAR, 2017,. All rights reserved.

\section{Introduction}

Among the hospital infections caused by resistant microorganisms, great difficulties are experienced in the treatment of the cases in which the agent is Acinetobacter baumannii. Dual combinations or colistin are included in the treatment of multidrug resistant (MDR) and extremely drug resistant (XDR) A. baumannii infections [1]. XDR A. 
baumannii is resistant to carbapenem and all other antibiotics except colistin and tigecycline. Colistin resistance is a severe problem that has confronted us lately [2].

A. baumannii is a bacterium that can keep its vitality for a long time in dry settings, dry weather and inanimate surfaces [3]. For this reason, it can cause severe infections in all settings of hospitals especially in intensive care units. Besides the periods in which the compliance with hand hygiene and standard isolation procedures decrease, application of mechanical ventilation to patients, surgical operation, application of invasive procedures, immunosuppressive treatment regimens and long stays give rise to an increase in MDR A. baumannii colonization and infection [4].

Ventilator-associated pneumonia (VAP) develops 48 hours or longer after mechanical ventilation. VAP is a serious clinical condition that can occur in patients who undergo mechanical ventilation. The agents are usually resistant microorganisms. They may be invasive from the hospital environment through the endotracheal tube to the respiratory system and lung parenchyma tissue.

For the infections of respiration, blood circulation, wound and urinary tract, the treatment options have become extremely restricted due to the recently- increased drug resistance [5]. The performed studies show that the common usage of wide-spectrum antibiotics such as cefalosporins and carbapenems play a role in the selection of MDR clones $[1,5]$. Treatment with colistin have recently been seen to come to forefront.

Our study aims to examine the factors affecting the morbidity and mortality together with the type and period of treatment applied for MDR and XDR A. baumannii that developed in intensive care unit patients.

\section{Material and Method}

Study Design and Population

A retrospective study was conducted at İzmir Menemen State Hospital, a 226-bed hospital in İzmir, Turkey with an annual average admission rate of 759.537 patients. The study was approved by the ethics committee in İzmir Katip Çelebi University at August, 132015.

From January 2014 to July 2015, there were 225 patients with suspected VAP in the ICU. In 83 cases, bacterial growth was observed in culture plate. Twenty of these growth were the A. baumannii as causative agents.

Patients with positive quantitative culture from low respiratory tract specimens (e.g., bronchoalveolar lavage and bronchial secretions, $\geq 10^{5}$ ) for A. baumannii identified between January 2014 and July 2015 were evaluated. Patients 22-90 years of age who received greater than 48 hours of treatment were included in the study. Pneumonia was considered VAP if the onset occurred $\geq 48$ hours after intubation and was diagnosed based on new or progressive pulmonary infiltrates on chest X-ray and two or more of the following: fever $>38^{\circ} \mathrm{C}$ or hypothermia $<35.5^{\circ} \mathrm{C}$, leukocytosis $>12,000$ cells $/ \mathrm{mL}$ or leukopenia $<4,000$ cells $/ \mathrm{mL}$, purulent bronchial secretions; and supported by the result of a qualitative culture test (positive for $A$. baumannii isolate)

\section{Organism Identification and Antimicrobial Susceptibility Testing}

Species identification to the level of $A$. baumannii complex was completed with gram staining, oxidase test and the API 20E strip (BioMerieux, France). Antimicrobial susceptibility testing was performed on all isolates by either Kirby-Bauer disk diffusion method (Mastdiscs, Mast Diagnostic, UK) and E test (BioMerieux, France) for colistin, meropenem and imipenem to determine minimum inhibitory concentrations (MICs). Antimicrobial susceptibilities were interpreted according to Clinical and Laboratory Standards Institute (CLSI) guidelines [6]. The antimicrobials tested were piperacillin/tazobactam $(100 / 10 \mu \mathrm{g})$, ampicillin/sulbactam $(10 \mu \mathrm{g})$, imipenem $(10 \mu \mathrm{g})$, meropenem $(10$ $\mu \mathrm{g})$, gentamicin $(10 \mu \mathrm{g})$, amikacin $(30 \mu \mathrm{g})$, ceftriaxone $(30 \mu \mathrm{g})$, ceftazidime $(30 \mu \mathrm{g})$, cefepime (30 $\mu \mathrm{g})$, ciprofloxacin $(5 \mu \mathrm{g})$, levofloxacin $(5 \mu \mathrm{g})$ and trimethoprim-sulfamethoxazol $(1.25 / 23.75 \mu \mathrm{g})$. For colistin, meropenem, imipenem, MICs were determined by E-test based on the manufacturer's recommendations (bioMerieux). Pseudomonas aeruginosa ATCC 27853 and Escherichia coli ATCC 25922 were used as control strains for susceptibility tests.

\section{Data Collection}

Inpatient electronic medical records, pharmacy databases, and clinical microbiology databases were screened for two years to reach data of patients. Recorded patient demographics included age, gender, and other patient 
comorbidities (diabetes, lung and cardiac dysfunction, and renal dysfunction). The class of active antibiotic, and total number of antibiotic days post culture was defined.

\section{Statistical Analysis}

The results were expressed as number of observations (n) and ratio (\%). Odds ratio (OR) values and their 95\% confidence intervals $(95 \% \mathrm{CI})$ were calculated. Statistical significance was set at $\mathrm{p} \leq 0.05$. All analysis was carried out using the Statistical Package for Social Sciences (SPSS) version 20.

\section{Results}

During the study period, a total of 20 cases with positive culture of $A$. baumannii were identified. The mean age was $71,9 \pm 16,4$ years, with a range of 22 to 90 years. All of cases were included because they met VAP criteria and the study inclusion criteria. All cases diagnosed with A. baumannii-VAP were caused by MDR- A. baumannii.

In the current study, most cases were patients admitted to the ICU due to pneumonia and cerebrovascular disease. Median days of stay in ICU was $40.8-/+20$ day. The values of Acute Physiology and Chronic Health Evaluation II (APACHE II) was 26,8 $\pm 3,2$.

Table 1 outlines detailed clinical characteristics of all cases included in the study. More than half of included patients $(65 \%, 13 / 20)$ had at least one co-morbid disease. Hypertension was also present in 7 of 13 patients who died. There was chronic renal failure in 5 case, and chronic obstructive pulmonary disease (COPD) in 6 case, also.

All patients received at least two drugs while they were receiving ICU. Empirical therapy was used in all patients $(100 \%)$, carbapenems and/or ceftriaxone were the most commonly used empirical agents $(75 \% ; 15 / 20)$, and colistin $(100 \% ; 20 / 20)$ was the most commonly used definitive antibiotic.

The 14-day mortality among patients infected with MDR- A. baumannii VAP was 65\% (13/20). The results of the univariate analysis of predictors of mortality are shown in Table 2 . There was no significant difference in the between age and gender variables on surviving group $(\mathrm{p}>0.05)$.

More cases in the non-survival group used hearth failure drugs, bronchodilators and vasopressors. Surviving 5 cases had used ipratropium/salbutamol nebulizer during therapy.

All of the cases had infection one or more different region of body. Blood cultures were detected positive in 8 patients. Additionally, A. baumannii was growth 2 patients blood culture. Other bacteria isolated from blood cultures of methicillin resistant $S$. aureus, methicillin resistant coagulase negative staphylococcus and $E$. coli has been identified. During periods of intensive care, infections with different microorganisms were also detected in the patients' urine, wound and sputum cultures.

There was a significant difference in the use of colistin alone and ampicillin/sulbactam combination from the colistin+carbapenem combination between the survival and nonsurvival groups $(\mathrm{p}<0.05)$.

Table 3 shows the results of antibiotic susceptibility assay for MDR-AB isolates. The isolates were tested against different types of antibiotics. No strain was sensitive to ceftriaxone, imipenem, ciprofloxacin, ampicillin/sulbactam, and piperacillin/tazobactam. Colistin was the most active agent $17 / 20$ ( $85 \%$ sensitive) followed by gentamicin $6 / 20$ (30\% sensitive) and trimethoprim/sulfametoxazole $4 / 20$ (20\% sensitive).

Table 1. Demographic and clinical characteristics of patients.

\begin{tabular}{|c|c|c|c|}
\hline & Male $\mathbf{n}(\%)$ & Female $\mathbf{n}(\%)$ & Total n(\%) \\
\hline Gender n (\%) & $10(50)$ & $10(50)$ & $20(100)$ \\
\hline Age (n; mean) & $10 ; 70,6$ & $10 ; 73,3$ & $20 ; 71,9$ \\
\hline Days of stay in ICU & $10 ; 40$ & $10 ; 41,6$ & $20 ; 40,8$ \\
\hline Primary Diagnosis & Single $* 15(75)$ & Multiply $* * 5(25)$ & $20(100)$ \\
\hline Nephrotoxicity & $15(75)$ & $5(25)$ & $20(100)$ \\
\hline Prognosis & $7(35)$ & $13(65)$ & $20(100)$ \\
\hline
\end{tabular}

*Single diagnosis: Chronic Obstructive Pulmoner Disease, Cardiac Failure and Cerebrovascular Disease 
**Comorbid disease: Hypertension at 7 cases and Chronic Renal Failure at 5 cases

Table 2: Analysis of predictors of mortality.

\begin{tabular}{|l|l|l|l|}
\hline & Cure n(\%) & Exitus n(\%) & Total n(\%) \\
\hline Female & $3(30)$ & $7(70)$ & $10(100)$ \\
\hline Male & $4(40)$ & $6(60)$ & $10(100)$ \\
\hline Nephrotoxicity & $1(20)$ & $4(80)$ & $5(100)$ \\
\hline Single disease & $7(46,7)$ & $8(53,3)$ & $15(100)$ \\
\hline Multiply disease & 0 & $5(100)$ & $5(100)$ \\
\hline Colistin & 0 & $4(100)$ & $4(100)$ \\
\hline Colistin+Carbapenem & $3(60)$ & $2(40)$ & $5(100)$ \\
\hline Colistin+Sulbactam & $4(36,4)$ & $7(63,6)$ & $11(100)$ \\
\hline
\end{tabular}

Table 3. The results of antibiotic susceptibility assay for multidrug-resistant A. baumannii isolates.

\begin{tabular}{|c|r|r|}
\hline \multirow{2}{*}{ Antibiotic } & \multicolumn{2}{|c|}{ Percentage of isolates (\%) } \\
\cline { 2 - 3 } & Resistant & Sensitive \\
\hline Amikacin & 100 & 0 \\
\hline Ampicillin/sulbactam & 100 & 0 \\
\hline Cefepime & 100 & 0 \\
\hline Ceftazidime & 100 & 0 \\
\hline Ceftriaxone & 100 & 0 \\
\hline Ciprofloxacin & 100 & 0 \\
\hline Colistin (E test) & 15 & 85 \\
\hline Gentamicin & 70 & 30 \\
\hline Imipenem (Disk diffusion and E test) & 100 & 0 \\
\hline Levofloxacin & 100 & 0 \\
\hline Meropenem (Disk diffusion and E test) & 100 & 0 \\
\hline Piperacillin/Tazobactam & 100 & 0 \\
\hline Trimethoprim-sulfamethoxazol & 80 & 20 \\
\hline
\end{tabular}

\section{Discussion:-}

A. baumannii isolates cause severe infections such as pneumonia, meningitis, bacteremia, show multiple resistance to antibiotics, give rise to hardships in treatment and progress with high mortality rates.

Inappropriate, frequent and long-run usage of wide-spectrum antibiotics cause resistant microorganisms to increase. Hospital infections that develop depending on resistant gram-negative microorganisms are one of the most important problems of ICUs. Kollef et al. reported that the mortality rate of $12.2 \%$ with proper antibiotic treatment for intensive care cases have risen up to $52.1 \%$ with improper antibiotic usage [7]. Acinetobacter species with the feature of developing multiple drug resistance give rise to life threatening infections. In the treatment of such infections, drug usage in high concentration, long-term infusion application, and reapplication of former antibiotics are some alternative methods. One of the former antibiotics that started to be reapplied is colistin (polymyxin E).

In Acinetobacter spp infections, many risk factors have been defined that cause mortality. Ventilator-associated pneumonia, urinary tract infection, wound infection and bacteremia depending on A. baumannii are nosocomial infections seen in intensive care units where invasive procedures are frequently applied [8]. Falagas et al. [9], in their studies, indicated that the mortality of $A$. baumannii infections ranged $7.8-23 \%$ in the cases staying in the services in hospitals, and 10-43\% in the cases in intensive care units. All of the patients included in our study are those of intensive care units and the mortality rate of the patients developing A. baumannii infection have been determined as $65 \%$.

In a study performed by Mulin et al. [10], it was detected that transferring patients to another unit is a risk factor for nosocomial colonization of A. baumannii. Ulu Kiliç et al. [11] did not find hospitalization directly to intensive care unit as a risk factor in terms of mortality, but found that transferring patient to more than one unit is a statistically significant risk factor. The reason for this is that the transferred patients are exposed to more cross contamination and infection-caught is more. Comorbidities and invasive procedures are seen more in these patients. In our study, it 
was detected that $8 / 20(40 \%)$ of the patients developing A. baumannii infection came from other services and $12 / 20$ $(60 \%)$ of them were directly hospitalized in intensive care unit.

Studies related to mortality associated with VAP due to A. baumannii are limited in the literature. GarnachoMontenero et al. [12] had found no differences between the two groups which are developed VAP related $A$. baumannii and other bacteria. Additionally, there was no difference between the two groups in terms of additional disease, age, sex, APACHE II score initially. However, they said that the length of hospitalization in pre-VAP period was a risk factor [12]. High APACHE II score was determined as factors affecting overall VAP mortality with elongation of hospitalization period in this study [12]. They did not find additional diseases associated with increased mortality. Furthermore, they did not observe a significant difference between imipenem sensitive and resistant A.baumannii related VAP mortality.

There are some studies showing that pneumonia mortality is associated with various additional diseases. Tejerina and colleagues [13] evaluated the risk factors for VAP development and mortality. The researchers found that the length of hospital stay was a risk factor for VAP development. The severity of the underlying disease, for example the presence of renal failure, shock table development, and hypoxemia were risk factors for mortality.

Diabetes mellitus (DM) has been found as a factor increasing mortality in most of the publications that examine the association of DM with pneumonia mortality [14]. Acute renal failure were also associated with mortality in patients with VAP [15]. Cardiac insufficiency, COPD, cerebrovascular disease, and immunosuppressive treatment were releated with mortality rate pneumonia in patients requiring hospital admission [16]. However; COPD, heart failure, renal insufficiency, DM presence were not associated with mortality in some studies [12]. In our study, a significant association with mortality rate was found in the patient group with hypertension (7/13 patient) ( $<<0.05)$. Additionally; heart failure, DM and COPD were not significantly higher in dead patients.

Şengül et al reported that there was a significant correlation $(\mathrm{p}<0.001)$ between APACHE II score and mortality in their studies [17]. In this study, they reported that renal failure releated with mortality, but DM not releated risk of mortality in VAP cases. When the additional diseases were evaluated in our study, there was no statistically significant relation with the presence of DM and COPD in the group with mortality. Renal function disorders due to hypertension are associated with mortality in our cases.

When the mortality-associated risk factors were evaluated by Edis et al, they reported that hypoalbuminemia, steroid use and the use of a mechanical ventilator increased mortality in A. baumannii pneumoniae [18]. If the patients did not receive appropriate antibiotic therapy, the mortality rate was reported to be 5-fold increased in this study.

In the studies in which risk factors were analyzed in Acinetobacter spp infection, there are several results about age. In a matched cohort study performed, old age was found out to be a significant risk factor for A.baumannii mortality [19].

In a study in which a univariate analysis is conducted in our country, being over the age of 50 was found to be significant for mortality, while 3.5 times as much death rate was detected in the patients over 50 as those not over 50 in a survival analysis [17]. Advanced age has been identified as an important risk factor in our study.

Blot et al. [20], in their case-control study in which they based on APACHE II score and hospitalization period in intensive care unit, did not find that the cited mortality was statistically significant. In our study, APACHE II score and hospitalization period in intensive care unit were not significant risk factors.

Ulu Kiliç et al [11] interpreted >16 APACHE II score as a significant risk factor in terms of mortality. In the end of the survival analysis, they found about three times as many death rates as the cases whose APACHE II scores was < 16.

In a study carried out in our country, the elongation of hospitalization period in hospital and in intensive care unit was detected to be a risk factor for colonization and infection [21]. In general, imipenem is a highly sensitive antibiotic for Acinetobacter spp. But, resistant strains are known to be gradually increasing [22]. Isolates resistant to imipenem are generally resistant to many drugs [3]. There is an increase in mortality rate and prolongation of hospital stay in A. baumannii infections with multiple antibiotic resistance. 
There are also studies examining the relationship between mortality and antibiotic resistance status of $A$. baumannii infections. In a study in which the prognosis of bacteremia caused resistant $A$. baumannii was investigated, a mortality of 30 days was reported to be $60.7 \%$ [22]. In another study in which A. baumannii bacteremia cases resistant to all antibiotics were assessed, mortality rate was found to be $60 \%$ and in the same study multiorgan dysfunction syndrome was reported to be an indicator in the estimation of mortality [23].

Regrettably, treatment options are limited of carbapenem resistant A. baumannii infections. The imipenem resistance in A. baumannii bacteremia is mostly the inappropriateness of the empirical treatment launced. Therefore, the effect of imipenem resistance on mortality was found to be significant [13]. In our study, imipenem resistance was determined to be $100 \%$. Carbapenem resistance in the cases who died was detected to be at a high rate like $100 \%$ $(13 / 13)$. It was found to be significant statistically $(\mathrm{p}<0.01)$. This high rate of carbapenem resistance can be attributed to the low number of patients in the study. In another study in which carbapenem resistance was not detected as a significant risk factor for mortality, this status was linked to the insufficiency of the number of patients and bacteremic episodes about catheter [12]. In another study in which suitable and unsuitable empirical antimicrobial treatment's effects on the mortality of the patients with A. baumannii infection were researched, cure rate in the group having had a suitable treatment was found to be significantly higher [24].

Clonal selection is also important in resistance to carbapenems, such as colistin resistance [25,26]. Nhu et al [27] indicated that a carbapenem-resistant A. baumannii clone had been introduced and then maintained on the ICU. They reported that surveillance of the aetiologies of VAP and their changing antimicrobial susceptibility profiles aids the development of appropriate management and treatment guidelines.

Suitable and early treatment was shown to reduce mortality depending on infection. Le Minh et al [28] reported that colistin resistance could develop in the first $24 \mathrm{~h}$ of treatment with colistin monotherapy, and that colistin resistance was suppressed and delayed by combination therapy. In this study, more carbapenem-resistant A. baumannii isolates exhibited synergistic activity with a combination of colistin and meropenem than carbapenem-susceptible $A$. baumannii isolates. According these results, combinations of colistin and meropenem should be considered when treating carbapenem-resistant $A$. baumannii infections.

Antimicrobial combinations are preferred in order to provide strong antimicrobial efficacy through synergistic effects. This kind of treatment applications allows minimizing resistant bacteria. Kiratisin et al reported that combinations of colistin with ceftazidime, ceftriaxone, imipenem or meropenem have in vitro synergistic activity against local carbapenem resistant A. baumannii strains in Vietnam. They found that colistin/meropenem combination had the greatest potential synergistic effect [29].

Aydemir et al [30] investigated that clinical, laboratory, radiological and microbiological response rates in 43 patient with VAP caused by a carbapenem-resistant $A$. baumannii strain. Their results suggest that the combination of colistin with rifampicin markedly shortened the healing process.

In our study, although antibiotics known to be active to A. baumannii were started such as colistin, ampicillin/sulbactam, and carbapenem the inappropriateness of the treatment of antibiotic which was being used or was empirically started was found to be $70 \%$ considering the resistance pattern, and it was found to be a significant risk factor for mortality. Choi et al [13] found the inappropriate empirical treatment to be significantly related to malign prognosis. The different results of these studies in which the appropriateness of empirical treatment was assessed can be caused by the fact that the evaluated populations had different antibiotic resistance patterns.

In conclusion, longer duration of ICU stay in the hospital in patients undergoing mechanical ventilation are important risk factors. In addition, broad spectrum antibiotics used in this process in intensive care patients lead to the selection of resistant bacteria [14].

Consequently, knowing the risk factors indicating the mortality in patients and early diagnosing the bacteremia by closely following the patients are of importance in terms of the prognosis of the patient. The effect of starting the appropriate treatment by making the correct decision about the severity of acute disease and underlying disease on the result in $A$. baumannii bacteremia will be better. Also, avoiding unnecessary antibiotic treatment and prophylaxis, applying strict infection control precautions and eradicating controllable risk factors can reduce potential bacteremia caused by A. baumannii and the deaths depending on them. 


\section{References:-}

1. Dijkshoorn L, Nemec A \& Seifert H. An increasing threat in hospitals: multidrug-resistant Acinetobacter baumannii. Nat Rev Microbiol 2007; 5: 939-51.

2. Peleg AY, Seifert H, Paterson DL. Acinetobacter baumannii: Emergence of a Successful Pathogen. Clin Microbiol Rev 2008; 21(3): 538-82.

3. Phumisantiphong U, Diraphat P, Utrarachkij F, Uaratanawong S, Siripanichgon K. Clonal spread of carbapenem resistant Acinetobacter baumannii in the patients and their environment at BMA Medical College and Vajira Hospital. J Med Assoc Thai 2009; 92 (7): 173-80.

4. Ece G, Erac B, Cetin HY, Ece C, and Baysak A. Antimicrobial Susceptibility and Clonal Relation Between Acinetobacter baumannii Strains at a Tertiary Care Center in Turkey. Jundishapur J Microbiol 2015; 8(2): e156122.

5. Kim YA, Choi JY, Kim CK, et al. Risk factors and outcomes of bloodstream infections with metallo-betalactamase-producing Acinetobacter. Scand J Infect Dis 2008; 40(3): 234-40.

6. Performance standards for antimicrobial susceptibility testing, Twenty-Fourth Informational Supplement 2014, M100-S24. Clinical and Laboratory Standards Institute, Wayne, PA.

7. Kollef MH, Sherman G, Ward S, Fraser VJ. Inadequate antimicrobial treatment of infections: a risk factor for hospital mortality among critically ill patients. Chest 1999; 115: 462-74.

8. Choi JY, Park YS, Kim CO, et al. Mortality risk factors of Acinetobacter baumannii bacteremia. Intern Med J 2005; 35(10): 599-603.

9. Falagas ME, Rafailidis PI. Attributable mortality of Acinetobacter baumannii: no longer a controversial issue. Crit Care 2007; 11(3): 134.

10. Mulin B, Talon D, Viel JF, et al. Risk factors for nosocomial colonization with multiresistant Acinetobacter baumannii. Eur J Clin Microbiol Infect Dis 1995; 14(7): 569-76.

11. Ulu Kiliç A, Ahmed SS, Alp E, Doğanay M. Challenge of intensive care unit-acquired infections and Acinetobacter baumannii in developing countries. OA Critical Care 2013; 1(1): 2.

12. Garnacho-Montero J, Ortiz-Leyba C, Fernández- Hinojosa E, et al. Acinetobacter baumannii ventilatorassociated pneumonia: epidemiological and clinical findings. Intensive Care Med 2005; 31: 649-55.

13. Tejerina E, Frutos-Vivar F, Restrepo MI, et al. Incidence, risk factors, and outcome of ventilator-associated pneumonia. J Crit Care 2006; 21: 56-65.

14. Gamble JM, Eurich DT, Marrie TJ, Majumdar SR. Admission hypoglycemia and increased mortality in patients hospitalized with pneumonia. Am J Med 2010; 123(6):556.e11-6. doi: 10.1016/j.amjmed.2009.11.021.

15. Inchai J, Pothirat C, Bumroongkit C, Limsukon A, Khositsakulchai W and Liwsrisakun C. Prognostic factors associated with mortality of drug-resistant Acinetobacter baumannii ventilator-associated pneumonia. J Intensive Care 2015; 3:9.

16. Bekaert M, Timsit JF, Vansteelandt S, et al. Attributable mortality of ventilator-associated pneumonia: a reappraisal using causal analysis. Am J Respir Crit Care Med 2011;184:1133-9.

17. Şengül A, Şengül E, Barış SA, Hayırlığlu N. Assesment of the factors associated with mortality in ventilator associated pneumonia of multidrug resistance Acinetobacter baumannii. Medical Journal of Kocaeli 2013; 1: 16

18. Edis EC, Hatipoglu ON, Tansel O, Sut N. Acinetobacter pneumonia: Is the outcome different from the pneumonias caused by other agents. Ann Thorac Med 2010; 5: 92-6.

19. Grupper M, Sprecher H, Mashiach T, Finkelstein R. Attributable mortality of nosocomial Acinetobacter bacteremia. Infect Control Hosp Epidemiol 2007; 28(3): 293-8.

20. Blot S, Vandewoude K, Colardyn F. Nosocomial bacteremia involving Acinetobacter baumannii in critically ill patients: a matched cohort study. Intensive Care Med 2003; 29(3): 471-5.

21. Alp E, Yerer M, Kocagöz S, et al. The risk factors and spread of multidrug-resistant Acinetobacter baumannii in intubated patients in a medical intensive care unit. Turk J Med Sci 2009; 39(5): 761-9.

22. Higgins PG, Dammhayn C, Hackel M, Seifert H. Global spread of carbapenem-resistant Acinetobacter baumannii. J Antimicrob Chemother 2010; 65(2):233-8. doi: 10.1093/jac/dkp428

23. Kim YJ, Kim SI, Kim YR, et al. Carbapenem-resistant Acinetobacter baumannii: diversity of resistant mechanisms and risk factors for infection. Epidemiol Infect 2012; 140: 137-45.

24. Tseng YC, Wang JT, Wu FL, Chen YC, Chie WC, Chang SC. Prognosis of adult patients with bacteremia caused by extensively resistant Acinetobacter baumannii. Diagn Microbiol Infect Dis 2007; 59(2): 181-90.

25. Kwon KT, Oh WS, Song JH, et al. Impact of imipenem resistance on mortality in patients with Acinetobacter bacteraemia. J Antimicrob Chemother 2007; 59(3): 525-30. 
26. Ahmed SS, Alp E, Ulu-Kilic, A. et al. Spread of carbapenem-resistant international clones of Acinetobacter baumannii in Turkey and Azerbaijan: a collaborative study. Eur J Clin Microbiol Infect Dis 2016; $35: 1463$. doi:10.1007/s10096-016-2685-x.

27. Nhu NT, Lan NP, Campbell JI, et al. Emergence of carbapenem-resistant Acinetobacter baumannii as the major cause of ventilator-associated pneumonia in intensive care unit patients at an infectious disease hospital in southern. Vietnam J Med Microbiol 2014; 63: 1386-94. doi:10.1099/jmm.0.076646-0.

28. Le Minh V, Thi Khanh Nhu N, Vinh Phat VV, et al. In vitro activity of colistin in antimicrobial combination against carbapenem-resistant Acinetobacter baumannii isolated from patients with ventilator-associated pneumonia in Vietnam. J Med Microbiol 2015; 64(10): 1162-9. doi:10.1099/jmm.0.000137.

29. Kiratisin P, Apisarnthanarak A. \& Kaewdaeng S. Synergistic activities between carbapenems and other antimicrobial agents against Acinetobacter baumannii including multidrugresistant and extensively drugresistant isolates. Int J Antimicrob Agents 2010; 36: 243-6.

30. Aydemir H, Akduman D, Piskin N, et al. Colistin vs. the combination of colistin and rifampicin for the treatment of carbapenem-resistant Acinetobacter baumannii ventilator-associated pneumonia. Epidemiol \& Infect 2013; 141 (6): 1214-22. 